VOLUME 22 (2017) 39-44

DOI: $10.24330 /$ ieja. 325920

\title{
A GG NOT FH SEMISTAR OPERATION ON MONOIDS
}

\author{
Ryûki Matsuda \\ Received: 17 September 2016; Revised: 27 March 2017 \\ Communicated by Sait Halıcıoğlu
}

\begin{abstract}
Let $S$ be a g-monoid with quotient group $\mathrm{q}(S)$. Let $\overline{\mathrm{F}}(S)$ (resp., $\mathrm{F}(S), \mathrm{f}(S)$ ) be the $S$-submodules of $\mathrm{q}(S)$ (resp., the fractional ideals of $S$, the finitely generated fractional ideals of $S)$. Briefly, set $\mathrm{f}:=\mathrm{f}(S), \mathrm{g}:=\mathrm{F}(S), \mathrm{h}:=$ $\overline{\mathrm{F}}(S)$, and let $\{\mathrm{x}, \mathrm{y}\}$ be a subset of the set $\{\mathrm{f}, \mathrm{g}, \mathrm{h}\}$ of symbols. For a semistar operation $\star$ on $S$, if $\left(E+E_{1}\right)^{\star}=\left(E+E_{2}\right)^{\star}$ implies $E_{1}{ }^{\star}=E_{2}{ }^{\star}$ for every $E \in \mathrm{x}$ and every $E_{1}, E_{2} \in \mathrm{y}$, then $\star$ is called xy-cancellative. In this paper, we prove that a gg-cancellative semistar operation need not be fh-cancellative.
\end{abstract}

Mathematics Subject Classification (2010): 13A15

Keywords: Semistar operation, monoid

\section{Introduction}

A subsemigroup $S \supsetneqq\{0\}$ of a torsion-free abelian additive group is called $a$ grading monoid (or, a g-monoid) (D. Northcott [12]). We will use, for g-monoids, the following terminologies: module, ideal, valuation, star operation analogously to them for rings (cf., [4]). Thus, let $S$ be a g-monoid, and let $X$ be a non-empty set. Assume that, for every $s \in S$ and $x \in X$, an element $s+x$ of $X$ is defined so that $0+x=x$ and, for every $s_{1}$ and $s_{2}$ in $S,\left(s_{1}+s_{2}\right)+x=s_{1}+\left(s_{2}+x\right)$, then $X$ is called a module over $S$ (or, an $S$-module). For the general theory of g-monoids, we refer to [5] and [7]. The additive group $\mathrm{q}(S):=\left\{s-s^{\prime} \mid s, s^{\prime} \in S\right\}$ is called the quotient group of $S$. Let $\overline{\mathrm{F}}(S)$ be the set of $S$-submodules of $\mathrm{q}(S)$. An element $E$ of $\overline{\mathrm{F}}(S)$ is called a fractional ideal of $S$ if $s+E \subseteq S$ for some $s \in S$. Let $\mathrm{F}(S)$ be the set of fractional ideals of $S$. A fractional ideal $I$ is called an ideal of $S$ if $I \subseteq S$. Let $\mathrm{f}(S):=\{E \in \mathrm{F}(S) \mid E$ is a finitely generated fractional ideal $\}$. A mapping $\star: \overline{\mathrm{F}}(S) \longrightarrow \overline{\mathrm{F}}(S), E \longmapsto E^{\star}$ is called a semistar operation on $S$ if it satisfies the following properties for every $x \in \mathrm{q}(S)$ and $E, F \in \overline{\mathrm{F}}(S)$ :

(1) $(x+E)^{\star}=x+E^{\star}$;

(2) $E \subseteq E^{\star}$ and $\left(E^{\star}\right)^{\star}=E^{\star}$;

(3) $E \subseteq F$ implies $E^{\star} \subseteq F^{\star}$. 
Set $\mathrm{f}:=\mathrm{f}(S), \mathrm{g}:=\mathrm{F}(S), \mathrm{h}:=\overline{\mathrm{F}}(S)$, and let $\{\mathrm{x}, \mathrm{y}\}$ be a subset of the set $\{\mathrm{f}, \mathrm{g}$, h\}. For a semistar operation $\star$ on $S$, if $\left(E+E_{1}\right)^{\star}=\left(E+E_{2}\right)^{\star}$ implies $E_{1}{ }^{\star}=E_{2}{ }^{\star}$ for every $E \in \mathrm{x}$ and $E_{1}, E_{2} \in \mathrm{y}$, then $\star$ is called $x y$-cancellative. A mapping $\star: \mathrm{F}(S) \longrightarrow \mathrm{F}(S), E \longmapsto E^{\star}$ is called a star operation on $S$ if it satisfies the following properties for every $x \in \mathrm{q}(S)$ and $E, F \in \mathrm{F}(S)$ :

(1) $S^{\star}=S$;

(2) $(x+E)^{\star}=x+E^{\star}$;

(3) $E \subseteq E^{\star}$ and $\left(E^{\star}\right)^{\star}=E^{\star}$;

(4) $E \subseteq F$ implies $E^{\star} \subseteq F^{\star}$.

We refer to M. Fontana and K. A. Loper [1] and F. Halter-Koch [6] for star and semistar operations and their Kronecker function rings.

The mapping $E \longmapsto \mathrm{q}(S)$ from $\overline{\mathrm{F}}(S)$ to $\overline{\mathrm{F}}(S)$ for every $E \in \overline{\mathrm{F}}(S)$ is a semistar operation, called the e-semistar operation on $S$. Also, a star (resp., semistar) operation $\star$ on $S$ is said to have finite type, if $E^{\star}=\bigcup\left\{F^{\star} \mid F \in \mathrm{f}(S)\right.$ with $\left.F \subseteq E\right\}$ for every $E \in \mathrm{F}(S)$ (resp., $E \in \overline{\mathrm{F}}(S)$ ). Let $\Gamma$ be a totally ordered abelian additive group, and let $v$ be a mapping from $\mathrm{q}(S)$ onto $\Gamma$. If $v(a+b)=v(a)+v(b)$ for every $a, b \in \mathrm{q}(S)$, then $v$ is called a valuation on $q(S)$. $\Gamma$ is called the value group of $v$, and the set $V:=\{a \in \mathrm{q}(S) \mid v(a) \geq 0\}$ is called the valuation semigroup belonging to $v$. If $V \supseteq S$, then $V$ is called a valuation oversemigroup of $S$.

Let $\left\{V_{\lambda} \mid \lambda \in \Lambda\right\}$ be a non-empty set of valuation oversemigroups of $S$. Then the mapping $E \longmapsto \bigcap_{\lambda \in \Lambda}\left(E+V_{\lambda}\right)$ from $\overline{\mathrm{F}}(S)$ to $\overline{\mathrm{F}}(S)$ is a semistar operation on $S$, called the semistar operation defined by $\left\{V_{\lambda} \mid \lambda \in \Lambda\right\}$. This semistar operation is fh-cancellative (cf., [4, Theorem 32.5]). If $\left\{V_{\lambda} \mid \lambda \in \Lambda\right\}$ is the set of all valuation oversemigroups of $S$, the semistar operation defined by the set is called the $b$ semistar operation on $S$.

In this paper, we prove that a gg-cancellative semistar operation need not be fh-cancellative.

\section{Preliminary results}

Various implications hold among the cancellation properties of semistar operations:

Proposition 2.1. ([3], [8], [9], [10], [11]) Let $\star$ be a semistar operation on a $g$ monoid $S$.

(1) $\star$ is hh-cancellative if and only if $\star$ coincides with the e-semistar operation. 
We have the following diagram of implications:

$$
\begin{aligned}
& \mathrm{hh}=\mathrm{hg}=\mathrm{hf} \Longrightarrow \mathrm{gh} \Longrightarrow \mathrm{gg} \Longrightarrow \mathrm{gf} \\
& \Downarrow \quad \Downarrow \quad \Downarrow \\
& \text { fh } \Longrightarrow \mathrm{fg} \Longrightarrow \mathrm{ff}
\end{aligned}
$$

(2) A gh-cancellative semistar operation of finite type need not be hh-cancellative. A gf-cancellative semistar operation of finite type need not be gg-cancellative. An fh-cancellative semistar operation of finite type need not be gf-cancellative. A gg-cancellative semistar operation of finite type need not be gh-cancellative.

(3) A gf-cancellative semistar operation need not be fg-cancellative.

Remark 2.2. ([2, Lemma 3]) We have a simplified diagram of implications in the case of finite type semistar operations:

$$
\mathrm{hh}=\mathrm{hg}=\mathrm{hf} \quad \Longrightarrow \mathrm{gh} \quad \Longrightarrow \text { gg } \Longrightarrow \mathrm{gf} \quad \Longrightarrow \text { fh }=\mathrm{fg}=\mathrm{ff} .
$$

\section{A gg not fh semistar operation}

Throughout the section, let $u_{1}, u_{2}, u_{3}, \cdots$ be an infinite set of indeterminates over a torsion-free abelian additive group $L$. Define $S_{0}:=\left\{a+k_{1} u_{1}+\cdots+k_{n} u_{n} \mid a \in\right.$ $L, 0 \leq k_{i} \in \mathbb{Z}$, and $\left.0<n \in \mathbb{Z}\right\}$. Then $S_{0}$ is a g-monoid, and $S_{0} \supseteq M:=$ $\left\{a+k_{1} u_{1}+\cdots+k_{n} u_{n} \mid k_{i}>0\right.$ for some $\left.i\right\}$ is the unique maximal ideal of $S_{0}$. Let $\mathrm{q}\left(S_{0}\right)$ be the quotient group of $S_{0}$. We have $\mathrm{q}\left(S_{0}\right)=\left\{a+l_{1} u_{1}+\cdots+l_{n} u_{n} \mid a \in L, l_{i} \in \mathbb{Z}\right\}$.

First, we review the following.

Lemma 3.1. ([11]) Let $S$ be a g-monoid, and let $S_{0}$ be the above g-monoid.

(1) For every $E \in \overline{\mathrm{F}}(S)$, we have $E^{b}=\{x \in \mathrm{q}(S) \mid n x \in n E$ for some positive integer $n\}$, where $n E:=\left\{x_{1}+\cdots+x_{n} \mid\right.$ every $\left.x_{i} \in E\right\}$.

(2) The b-semistar operation on $S$ has finite type.

(3) On $S_{0}$, the b-semistar operation is gg-cancellative and not gh-cancellative.

Lemma 3.2. ([4, Proposition (32.4)]) Let $\mathcal{S}$ be a subset of $\overline{\mathrm{F}}(S)$ with $\mathcal{S} \ni \mathrm{q}(S)$ such that, for every $x \in \mathrm{q}(S)$ and every $E \in \mathcal{S}, x+E \in \mathcal{S}$. For every $H \in \overline{\mathrm{F}}(S)$, set $H^{\star}:=\bigcap\{E \in \mathcal{S} \mid E \supseteq H\}$. Then the mapping $H \longmapsto H^{\star}$ is a semistar operation on $S$.

The semistar operation $\star$ in Lemma 3.2 is said to be defined by the set $\mathcal{S}$. Let $H_{0}:=\left(u_{1}-u_{2}, u_{2}-u_{3}, \cdots\right)$, and let $F_{0}:=\left(u_{1}, u_{2}\right)$, where, for a subset $X \subseteq \mathrm{q}\left(S_{0}\right),(X)$ denotes the $S_{0}$-submodule of $\mathrm{q}\left(S_{0}\right)$ generated by $X$. Let $\mathcal{S}:=$ 
$\left\{G^{b}, x+H_{0}{ }^{b}, \mathrm{q}\left(S_{0}\right) \mid G \in \mathrm{F}\left(S_{0}\right), x \in \mathrm{q}\left(S_{0}\right)\right\}$, and let $\star$ be the semistar operation on $S_{0}$ defined by $\mathcal{S}$.

Let $v$ be a valuation on $\mathrm{q}\left(S_{0}\right)$ non-negative on $S_{0}$ with value group $\Gamma$. Let $v\left(u_{i}\right)=\gamma_{i}$ for every $i$. Then we denote $v=v\left\langle u_{1}, u_{2}, u_{3}, \cdots\right\rangle=\left\langle\gamma_{1}, \gamma_{2}, \gamma_{3}, \cdots\right\rangle$.

Lemma 3.3. We have the following:

(1) $F_{0} \subseteq\left(u_{1}, u_{2}, u_{3}, \cdots\right) \subseteq H_{0}$, and $H_{0} \notin \mathrm{F}\left(S_{0}\right)$.

(2) $0 \notin H_{0}^{b}$.

Proof. (1) For every $i$, we have $u_{i}=u_{i+1}+\left(u_{i}-u_{i+1}\right) \in S_{0}+H_{0} \subseteq H_{0}$. Suppose that $H_{0} \in \mathrm{F}\left(S_{0}\right)$. Then there is $x \in \mathrm{q}\left(S_{0}\right)$ such that $x+H_{0} \subseteq S_{0}$. We may set $x=l_{1} u_{1}+\cdots+l_{n} u_{n}$ with every $l_{i} \in \mathbb{Z}$. We have $u_{n}-u_{n+1} \in H_{0}$, hence $x+u_{n}-u_{n+1} \in S_{0}$, hence $l_{1} u_{1}+\cdots+l_{n} u_{n}+u_{n}-u_{n+1} \in S_{0}$; a contradiction.

(2) Let $\left\{\alpha_{i} \mid i=1,2, \cdots\right\}$ be a set of positive real numbers such that $\alpha_{i}>\alpha_{i+1}$ for every $i$. Define the valuation $v$ (and its valuation oversemigroup $V$ ) to the real numbers $\mathbb{R}$ by $\left.v=v<u_{1}, u_{2}, \cdots\right\rangle=\left\langle\alpha_{1}, \alpha_{2}, \cdots\right\rangle$. Then $0 \notin H_{0}+V$, hence $0 \notin H_{0}{ }^{b}$.

Lemma 3.4. We have the following:

(1) We have $H_{0}{ }^{\star}=H_{0}{ }^{b}$, hence $0 \notin H_{0}{ }^{\star}$.

(2) We have $G^{\star}=G^{b}$ for every $G \in \mathrm{F}\left(S_{0}\right)$.

Proof. (1) Set $\left\{x_{\sigma} \mid \sigma \in \Sigma\right\}:=\left\{x \in \mathrm{q}\left(S_{0}\right) \mid H_{0} \subseteq x+H_{0}{ }^{b}\right\}$. Since $H_{0} \notin \mathrm{F}\left(S_{0}\right)$ by Lemma 3.3(1), there is no element $G \in \mathrm{F}\left(S_{0}\right)$ such that $H_{0} \subseteq G^{b}$. Hence $H_{0}{ }^{\star}=\bigcap_{\sigma}\left(x_{\sigma}+H_{0}{ }^{b}\right)$. Since $H_{0} \subseteq x_{\sigma}+H_{0}{ }^{b}$, we have $H_{0}{ }^{b} \subseteq\left(x_{\sigma}+H_{0}{ }^{b}\right)^{b}=$ $x_{\sigma}+\left(H_{0}{ }^{b}\right)^{b}=x_{\sigma}+H_{0}{ }^{b}$. It follows that $H_{0}{ }^{\star}=H_{0}{ }^{b}$.

(2) Set $\left\{G_{\lambda} \mid \lambda \in \Lambda\right\}:=\left\{G^{\prime} \in \mathrm{F}\left(S_{0}\right) \mid G \subseteq G^{\prime b}\right\}$, and set $\left\{x_{\sigma} \mid \sigma \in \Sigma\right\}:=$ $\left\{x \in \mathrm{q}\left(S_{0}\right) \mid G \subseteq x+H_{0}{ }^{b}\right\}$. Then $G^{\star}=\bigcap_{\lambda} G_{\lambda}{ }^{b} \bigcap_{\sigma}\left(x_{\sigma}+H_{0}{ }^{b}\right)$. Since $G \subseteq G_{\lambda}{ }^{b}$, we have $G^{b} \subseteq\left(G_{\lambda}{ }^{b}\right)^{b}=G_{\lambda}{ }^{b}$, hence $\bigcap_{\lambda} G_{\lambda}{ }^{b}=G^{b}$. Since $G \subseteq x_{\sigma}+H_{0}{ }^{b}$, we have $G^{b} \subseteq\left(x_{\sigma}+H_{0}{ }^{b}\right)^{b}=x_{\sigma}+\left(H_{0}{ }^{b}\right)^{b}=x_{\sigma}+H_{0}{ }^{b}$, i.e., $G^{b} \subseteq \bigcap_{\sigma}\left(x_{\sigma}+H_{0}{ }^{b}\right)$. It follows that $G^{\star}=G^{b}$.

Lemma 3.5. $\star$ is a gg-cancellative semistar operation on $S_{0}$.

Proof. Let $G \in\left(G+G^{\prime}\right)^{\star}$, where $G, G^{\prime} \in \mathrm{F}\left(S_{0}\right)$. By Lemma 3.4(2), we have $G \subseteq\left(G+G^{\prime}\right)^{b}$. By Lemma 3.1(3), we have $0 \in\left(G^{\prime}\right)^{b}$, hence $0 \in\left(G^{\prime}\right)^{\star}$.

Lemma 3.6. Let $F_{0}+H_{0} \subseteq x+H_{0}{ }^{b}$, and let $x=l_{1} u_{1}+l_{2} u_{2}+\cdots+l_{n} u_{n}$ with every $l_{i} \in \mathbb{Z}$. Then we have 
(1) Every $l_{i} \leq 0$.

(2) $\left(F_{0}+H_{0}\right)^{\star}=H_{0}{ }^{b}$.

Proof. (1) Suppose the contrary. There are the following cases.

(i) The case $p_{1}:=l_{1}>0$. We have $-x+F_{0}+H_{0} \subseteq H_{0}{ }^{b}$. Define the valuation $v$ (and its valuation oversemigroup $V$ ) to $\mathbb{Z}$ by $\left.v=v<u_{1}, u_{2}, u_{3}, \cdots\right\rangle=<$ $1,0,0, \cdots>$. Then we have $\left(-p_{1} u_{1}-l_{2} u_{2}-\cdots-l_{n} u_{n}\right)+\left(u_{2}\right)+\left(u_{4}\right) \in H_{0}{ }^{b} \subseteq H_{0}+V$, hence $-p_{1} \geq \min v\left(H_{0}\right)=0$; a contradiction.

(ii) The case $p_{2}:=l_{2}>0$. We have $-x+F_{0}+H_{0} \subseteq H_{0}{ }^{b}$. Define the valuation $v$ (and its valuation oversemigroup $V$ ) to $\mathbb{Z}$ by $\left.v=v<u_{1}, u_{2}, u_{3}, u_{4}, \cdots\right\rangle=<$ $0,1,0,0, \cdots>$. Then we have $\left(-l_{1} u_{1}-p_{2} u_{2}-\cdots-l_{n} u_{n}\right)+\left(u_{1}\right)+\left(u_{1}-u_{2}\right) \in H_{0}{ }^{b} \subseteq$ $H_{0}+V$, hence $-p_{2}-1 \geq \min v\left(H_{0}\right)=-1$; a contradiction.

(iii) The case $p_{a}:=l_{a}>0$ for some $a \geq 3$. We have $-x+F_{0}+H_{0} \subseteq H_{0}{ }^{b}$. Define the valuation $v$ (and its valuation oversemigroup $V$ ) to $\mathbb{Z}$ by $v=v<$ $\left.u_{1}, u_{2}, \cdots, u_{a}, u_{a+1}, \cdots\right\rangle=\langle 0,0, \cdots, 1,0, \cdots\rangle$. Then we have $\left(-l_{1} u_{1}-l_{2} u_{2}-\right.$ $\left.\cdots-p_{a} u_{a}-\cdots\right)+\left(u_{1}\right)+\left(u_{a-1}-u_{a}\right) \in H_{0}{ }^{b} \subseteq H_{0}+V$, hence $-p_{a}-1 \geq \min$ $v\left(H_{0}\right)=-1 ;$ a contradiction.

(2) Set $\left\{x_{\lambda} \mid \lambda \in \Lambda\right\}:=\left\{x \in \mathrm{q}\left(S_{0}\right) \mid F_{0}+H_{0} \subseteq x+H_{0}{ }^{b}\right\}$. By Lemma 3.3(1), we have $\left(F_{0}+H_{0}\right)^{\star}=\bigcap_{\lambda}\left(x_{\lambda}+H_{0}{ }^{b}\right)$. By (1), we have $-x_{\lambda} \in S_{0}$ for every $\lambda$, hence $H_{0}{ }^{b} \subseteq x_{\lambda}+H_{0}{ }^{b}$. It follows that $\left(F_{0}+H_{0}\right)^{\star}=H_{0}{ }^{b}$.

Lemma 3.7. The semistar operation $\star$ on $S_{0}$ is not fh-cancellative.

Proof. By Lemma 3.6(2), we have $F_{0} \subseteq\left(F_{0}+H_{0}\right)^{\star}$. On the other hand, by Lemma 3.4(1), we have $0 \notin H_{0}{ }^{\star}$.

Lemma 3.5 and Lemma 3.7 imply the following.

Proposition 3.8. A gg-cancellative semistar operation need not be fh-cancellative.

We finish with an easy note.

Remark 3.9. We have that $S_{0}^{\star}=S_{0}, M^{\star}=M$, and that $\star$ is not of finite type. Further, the restriction of $\star$ to $\mathrm{F}\left(S_{0}\right)$ is a star operation on $S_{0}$.

\section{References}

[1] M. Fontana and K. A. Loper, Kronecker function rings: a general approach, Ideal theoretic methods in commutative algebra (Columbia, MO, 1999), Lecture Notes in Pure and Appl. Math., 220 (2001), 189-205. 
[2] M. Fontana and K. A. Loper, Cancellation properties in ideal systems: A classification of e.a.b. semistar operations, J. Pure Appl. Algebra, 213(11) (2009), 2095-2103.

[3] M. Fontana, K. A. Loper and R. Matsuda, Cancellation properties in ideal systems: an e.a.b. not a.b. star operation, Arab. J. Sci. Eng. ASJE. Math., 35 (2010), 45-49.

[4] R. Gilmer, Multiplicative Ideal Theory, Pure and Applied Mathematics, 12, Marcel Dekker, Inc., New York, 1972.

[5] R. Gilmer, Commutative Semigroup Rings, Chicago Lectures in Mathematics, University of Chicago Press, Chicago, IL, 1984.

[6] F. Halter-Koch, Ideal Systems: An Introduction to Multiplicative Ideal Theory, Monographs and Textbooks in Pure and Applied Mathematics, 211, Marcel Dekker, Inc., New York, 1998.

[7] R. Matsuda, Multiplicative Ideal Theory for Semigroups, 2nd ed., Kaisei, Tokyo, 2002.

[8] R. Matsuda, Note on g-monoids, Math. J. Ibaraki Univ., 42 (2010), 17-41.

[9] R. Matsuda, Cancellation properties in ideal systems of monoids, Int. Electron. J. Algebra, 9 (2011), 61-68.

[10] R. Matsuda, Note on cancellation properties in ideal systems, Comm. Algebra, 43(1) (2015), 23-28.

[11] R. Matsuda, A gg not gh semistar operation on monoids, Bull. Allahabad Math. Soc., 31(1) (2016), 111-119.

[12] D. G. Northcott, Lessons on Rings, Modules and Multiplicities, Cambridge Univ. Press, London, 1968.

Ryûki Matsuda

2241-42 Hori, Mito

Ibaraki 310-0903, JAPAN

email:rmazda@adagio.ocn.ne.jp 